\title{
Nano Meter Stepping Drive of Surface Acoustic Wave Motor
}

\author{
Takashi Shigematsu*, Minoru Kuribayashi Kurosawa*, and Katsuhiko Asai** \\ *Tokyo Institute of Technology, Department of Advanced Applied Electronics, \\ G2-606, 4259 Nagatsuta, Midori-ku, Yokohama, 226-8502, Japan \\ tucker@ae.titech.ac.jp,mkur@ae.titech.ac.jp \\ **Matsushita Electric Industrial Co., Ltd., Advanced Technology Research Laboratories, \\ 3-4 Hikaridai, Seika, Souraku, Kyoto 619-0237, Japan \\ kasai@crl.mei.co.jp
}

\begin{abstract}
A high resolution, from nanometer to sub-nanometer, stepping drive of a surface acoustic wave (SAW) motor is presented in this paper. A step displacement was controlled easily by a number of driving waves from centimeter order to nanometer order. Reduction of the driving waves down to 25 cycles, at 100 Vpeak driving voltage and $30 \mathrm{~N}$ pre-load condition, brought about 2 $\mathrm{nm}$ stepping drive with our experimental setup at open loop condition. We also demonstrated a sub-nanometer stepping drive. These experimental results indicate that the surface acoustic wave motor has an ability of subnanometer positioning with centimeter stroke.
\end{abstract}

\section{Introduction}

An ultrasonic motor is a motor which uses ultrasonic vibration-a type of elastic vibration- to obtain a driving force, which then drives the motor using friction [1]. Surface acoustic wave motor is a kind of ultrasonic motor that uses $9.6 \mathrm{MHz}$ Rayleigh wave, a kind of surface acoustic wave, for friction drive.

Conventional ultrasonic motors, which use micrometer order vibration for friction drive, have sub-micrometer positioning [1]. Surface acoustic wave motor uses nanometer order vibration for friction drive. On the analogy of conventional ultrasonic motors, we have suggested surface acoustic wave motor's possibility of

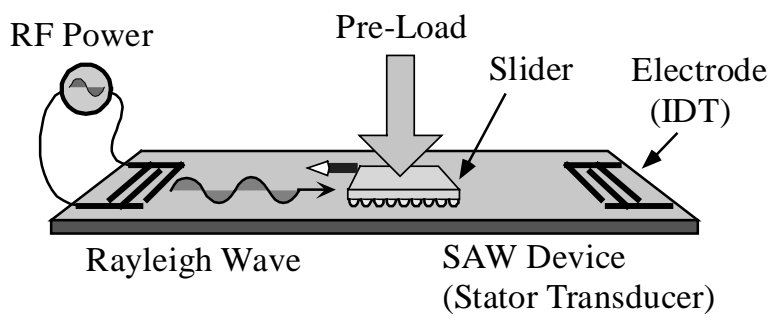

Fig.1 Schematic view of a surface acoustic wave motor. nanometer order stepping drive [2][3]. Due to the technical limit of measuring equipments, we have only reported $40 \mathrm{~nm}$ [4] and $25 \mathrm{~nm}$ [5] stepping drives. This paper describes the stepping drives of surface acoustic wave motor, down to sub-nanometer order, measured with a laser Doppler vibrometer that has $2 \mathrm{pm}$ resolution.

\section{Principle}

A schematic view of a surface acoustic wave motor is illustrated in Fig.1. A surface acoustic wave device (stator transducer) is made of $127.8^{\circ}$ y-rotated $\mathrm{x}$ propagating $\mathrm{LiNbO}_{3}$ substrate. RF electrical power (9.6 $\mathrm{MHz}$ ) is transduced to Rayleigh wave at the interdigital transducer (IDT) with piezoelectric effect. Surface particles of propagating Rayleigh wave move in elliptical motion, as illustrated in Fig.2. This kind of particles' elliptical motion is utilized for conventional traveling wave type ultrasonic motors.

However, the vibration amplitude of a surface acoustic wave device is extremely smaller, about $20 \mathrm{~nm}$ with $100 \mathrm{~V}$ peak driving voltage, due to the high driving frequency. The elliptical motion of surface particles yields friction driving force with a slider surface. A slider is made of silicon substrate being processed with

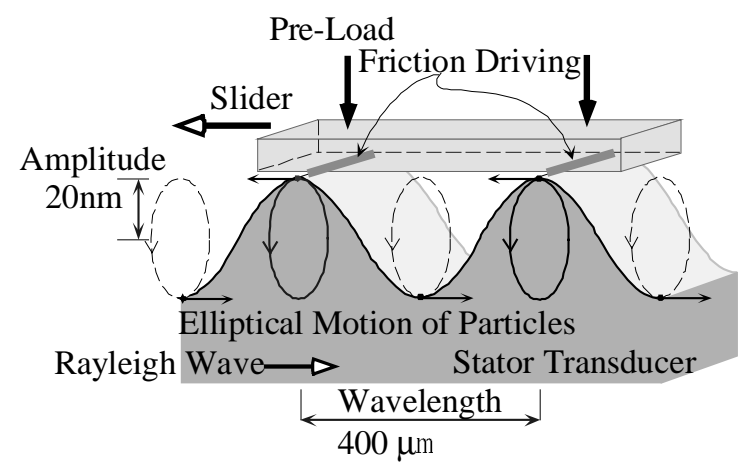

Fig. 2 Principle of a surface acoustic wave motor. 
surface micro fabrication technology to obtain output mechanical power efficiently [6][7]. Pre-load of the slider that means pushing the slider against the stator transducer enlarges friction force between the stator transducer and the slider. The large frictional force brings about large output force of the motor.

\section{Experimental Setup}

An experimental setup is shown in Fig.3. The setup is consists of a fixed part and a movable part. A $60 \times 15 \times 1$ $\mathrm{mm}^{3}$ stator transducer and a linear guide rail are the fixed part, and they are fixed in parallel. At each end of the stator transducer, inter digital transducers (IDTs) are deposited. The dimensions of the IDT are $400 \mu \mathrm{m}$ in pitch, $100 \mu \mathrm{m}$ in electrode strip width and $9 \mathrm{~mm}$ in aperture size, as illustrated in Fig.4. The IDT is composed of 20 strip electrode pairs and the resonance frequency for Rayleigh wave propagation is $9.6 \mathrm{MHz}$. A movable part comprises a $4 \times 4 \times 0.3 \mathrm{~mm}^{3}$ silicon slider, a steel hemisphere and a linear guide stage. The movable part moves along the linear guide rail. The silicon slider is glued on the steel hemisphere. The steel hemisphere is connected with the linear guide stage so that the silicon slider contacts a stator transducer surface properly. The movable part is schematically illustrated in Fig.5. A laser beam of the laser Doppler vibrometer was incident on the steel hemisphere to measure the step motion of the silicon slider. The pre-load is given by a coil spring so that the pre-load adjusted easily.

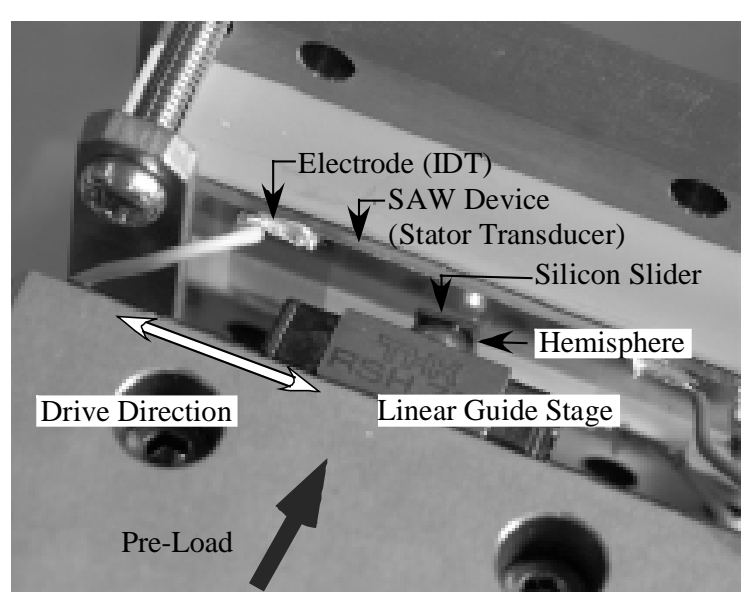

Fig.3 Photograph of the experimental setup.
Burst driving voltages were applied to the IDT every $0.2 \mathrm{~ms}$ to drive the surface acoustic motor in stepping motion. The waveform of the applied burst driving voltage is schematically shown in Fig.6. An example of stepping drive motion is shown in Fig.7, where the driving voltage was $100 \mathrm{~V}$ peak, the driving waves were 50 cycles, and the pre-load was $30 \mathrm{~N}$. Step motions were observed every $0.2 \mathrm{~ms}$ when the driving voltages were applied. Each step displacement was about $20 \mathrm{~nm}$.

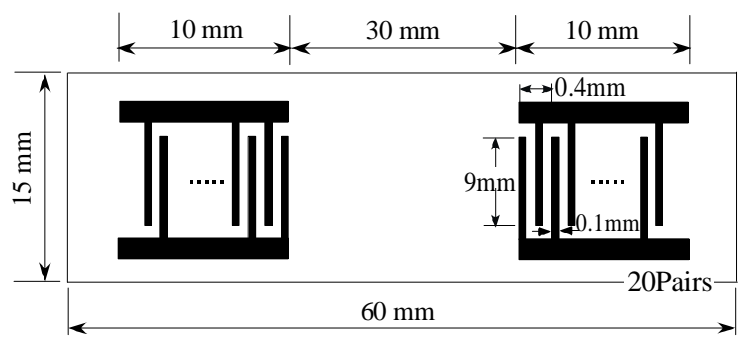

Fig.4 Geometry of the stator transducer and IDTs.

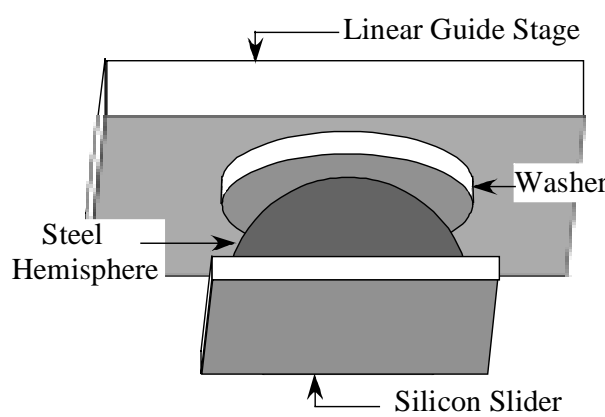

Fig.5 Schema of the driving part of the setup.

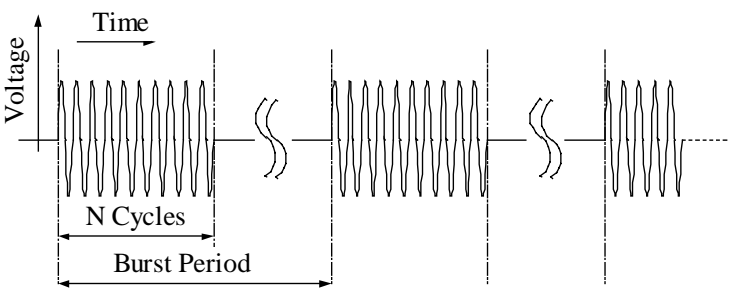

Fig.6 Waveform of the driving voltage. 


\section{Characteristics of Stepping Drive}

Figure 8 shows stepping drive motion in detail. This motion is the same motion of Fig.7, but it is enlarged to examine the detailed step motion. Two parts composed a single step, where the driving voltage was applied; the Rayleigh wave was excited, and vice versa. When the Raylegih wave is excited, friction with surface particles of the stator transducer yields the driving force to the silicon slider. The friction driven displacement is defined in Fig.8, as the displacement during the driving voltage is applied. It is the first part of the step motion and is the actual driven displacement due to the friction between the stator transducer and the silicon slider.

The second part is where the driving voltage is not applied; the Rayleigh wave has passed away. There is no driving force to the silicon slider at this part, but extra displacement seems to be yielded in Fig.8. It was caused by the inertia of the linear guide stage. The mass of the linear guide stage was $9.42 \mathrm{~g}$, and the one of the silicon slider with glued hemisphere was $0.89 \mathrm{~g}$. The mass of the linear guide stage was much larger and the friction with the linear guide rail was existed. Then the response of the linear guide stage was slower. Hence, the silicon slider (and the glued hemisphere) was dragged when the Rayleigh wave had passed away. It indicated that the movable part of the experimental setup did not move as a single mass. Namely, the movable part behaved as the two connected masses. One was the lighter slider part that consisted of the silicon slider and the steel hemisphere, and the other was the heavier linear guide stage part.

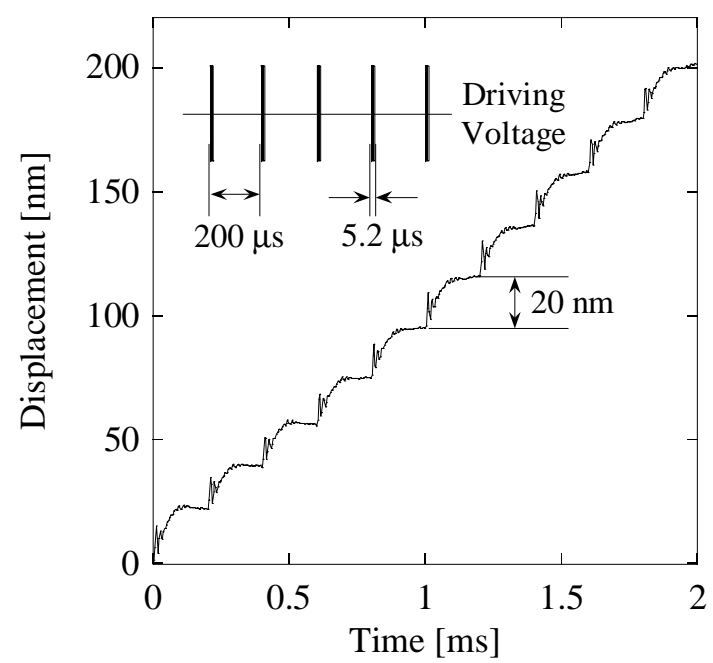

Fig.7 The stepping drive motion; the driving voltage was $100 \mathrm{~V}$ peak, and the driving waves were 50 cycles, and the pre-load was $30 \mathrm{~N}$. The burst period was $0.2 \mathrm{~ms}$.
After the friction drive, vibration occurred as shown in Fig.8. This vibration happened at the connection of the movable part's two masses. The washer and the steel hemisphere were used for the connection of these two parts to make contact the silicon slider against the stator transducer properly. The friction drive of the silicon slider makes elastic deformation at this connected region during the first part of the step motion. The elastic deformation causes the followed vibration of the second part of the step motion.

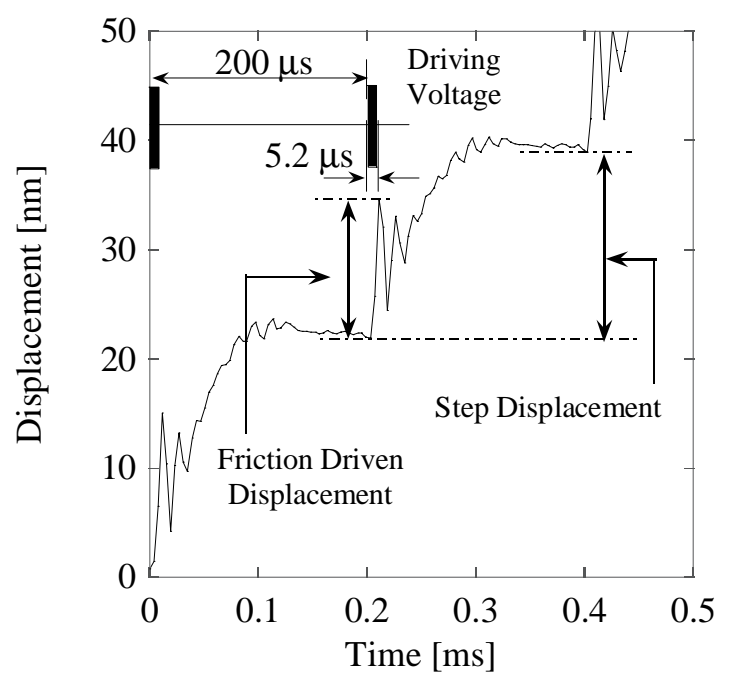

Fig.8 Detail of the stepping drive; the driving voltage was 100 Vpeak, the driving waves were 50 cycles, and the pre-load was the $30 \mathrm{~N}$. The burst period was $0.2 \mathrm{~ms}$. It is the same condition of the Fig.7.

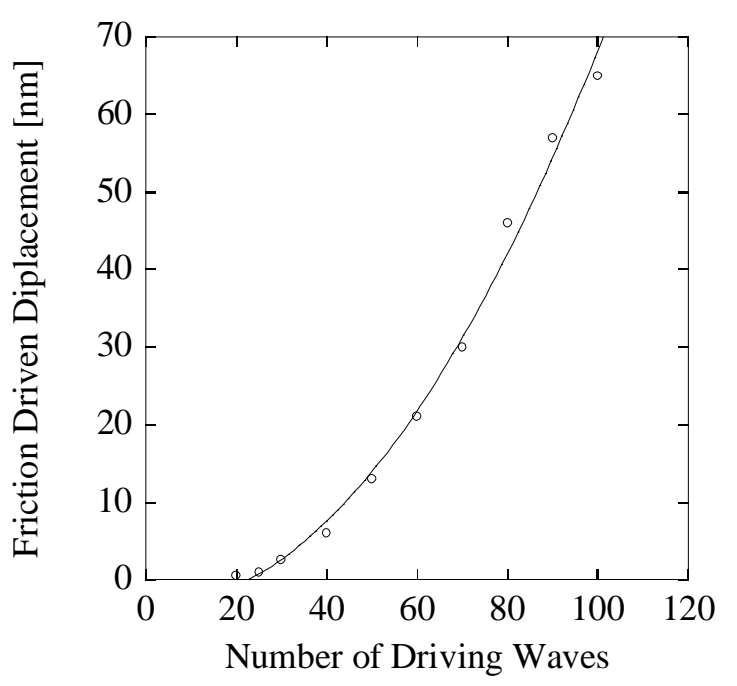

Fig.9 Friction driven displacement against the number of driving waves; the driving voltage was $100 \mathrm{~V}$ peak, and the pre-load was $30 \mathrm{~N}$. 
The friction driven displacements of stepping drives were measured for estimation of the friction drive, instead of the step displacement. The driving waves were varied from 20 cycles to 100 cycles, while the driving voltage was $100 \mathrm{~V}_{\text {peak, }}$ and the pre-load was 30 $\mathrm{N}$. The friction driven displacement against the number of the driving waves is shown in Fig.9. The friction driven displacement increased with the square of the number of the driving waves. The friction driven displacement of $0.5 \mathrm{~nm}$ was obtained at the 20 cycle driving waves.

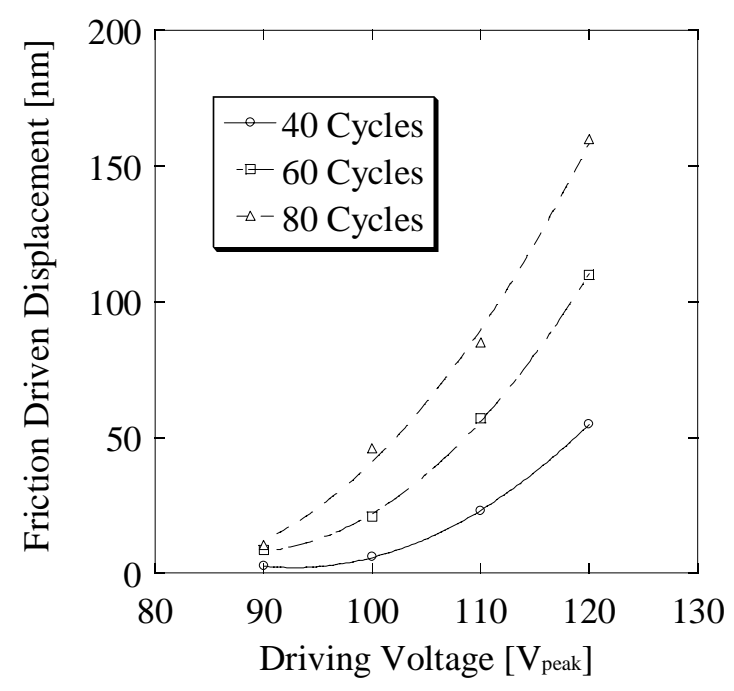

Fig.10 Friction driven displacement against the driving voltage; the driving waves were 40, 60, and 80 cycles, and the pre-load was $30 \mathrm{~N}$.

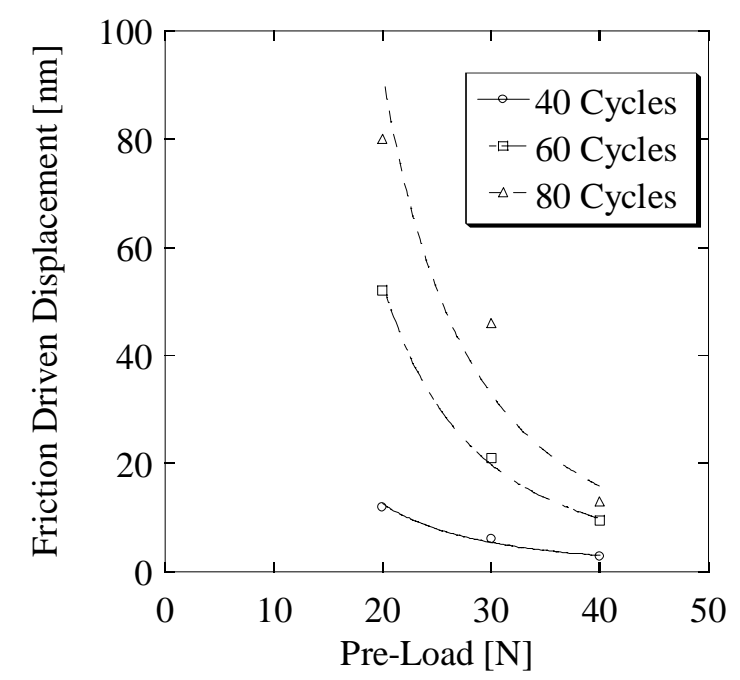

Fig.11 Friction driven displacement against the pre-load; the driving voltage was $100 \mathrm{~V}$ peak, and the driving waves were 40,60 , and 80 cycles.
The driving voltage was varied from $90 \mathrm{~V}$ peak to 120 $\mathrm{V}_{\text {peak, while the driving waves were } 40,60 \text { and } 80}$ cycles, and the pre-load was $30 \mathrm{~N}$. The friction driven displacement against the driving voltage is shown in Fig.10. The friction driven displacement increased with the square of the driving voltage at each driving waves. The pre-load was varied from $20 \mathrm{~N}$ to $40 \mathrm{~N}$, while the driving voltage was $100 \mathrm{~V}$ peak, and the driving waves were 40, 60 and 80 cycles. The friction driven displacement against the pre-load is shown in Fig.11. The friction driven displacement decreased in inverse proportion to the pre-load.

\section{Nano Meter Stepping Drive}

Changing of the number of the driving waves is the easiest way to obtain desired stepping drive at open loop condition. The friction driven displacement increased with the square of the number of the driving waves as previously shown in Fig.9. Then, the nanometer order stepping drives were examined with reduction of the driving waves, while the driving voltage and the pre-load were maintained at $100 \mathrm{~V}$ peak and $30 \mathrm{~N}$. With the 40 cycles of the driving waves brought about $8 \mathrm{~nm}$ stepping drive as illustrated in Fig.12. It was the finest stepping drive we obtained without signal processing of the measured motion into the less noise data signal. The drift of the laser Doppler vibrometer deserved about 5 to $10 \mathrm{~nm}$, then it was uncertain to obtain the stepping drive motion under 5 $\mathrm{nm}$ with single shot of measurement.

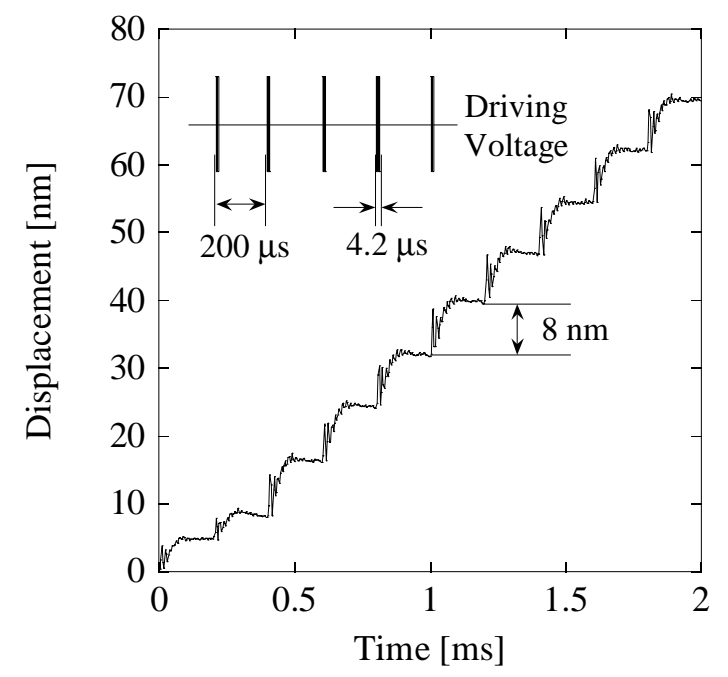

Fig.12 The $8 \mathrm{~nm}$ stepping drive; the driving voltage was $100 \mathrm{~V}$ peak, the driving waves were 40 cycles, and the pre-load was $30 \mathrm{~N}$. The burst period was $0.2 \mathrm{~ms}$. 
Figure 13 shows the stepping drive motion with 25 driving waves, as the driving voltage was $100 \mathrm{~V}$ peak and the pre-load was $30 \mathrm{~N}$. It is the averaged motion of 28 times stepping drives. The step displacement was about $2 \mathrm{~nm}$, and the friction driven displacement was about $1 \mathrm{~nm}$. It was the finest stepping drive of the experimental setup. It seems that the inertia and the vibrations due to the elastic deformation were significant in these nanometer range of stepping drives. The beginning several cycles of the vibration followed after the friction drive had almost same amplitude of the friction driven displacement that was about $1 \mathrm{~nm}$.

The vibration becomes a problem at the sub-nanometer range stepping drive as shown in Fig.14. This graph shows the stepping drive motion with 20 driving waves, while the driving voltage was $100 \mathrm{~V}$ peak, and the preload was $30 \mathrm{~N}$. It is also the averaged motion of 30 times stepping drives. Mean traveling motion of $0.5 \mathrm{~nm}$ in each step with sub-nanometer vibration was observed. The friction driven displacement and the vibration amplitude caused by the elastic deformations were similar quantity at all the time of the stepping motion. This motion is no more called stepping drive, but $0.5 \mathrm{~nm}$ friction driven displacement was verified. Namely, the sub-nanometer step motion was masked with the vibration due to the compliance of the structure.

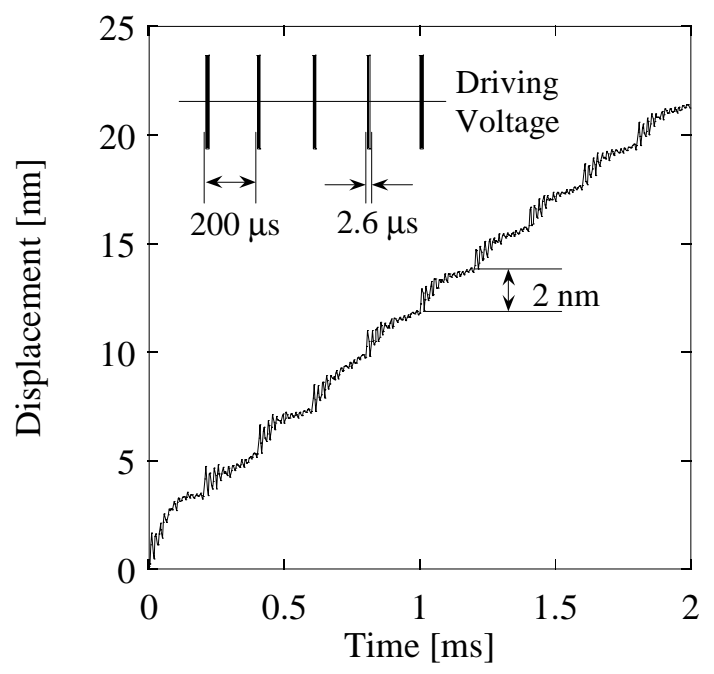

Fig.13 The finest stepping drive, $2 \mathrm{~nm}$ step, motion of the experimental setup; the driving voltage was 100 Vpeak, the pre-load was $30 \mathrm{~N}$, and the driving waves were 25 cycles. The burst period was $0.2 \mathrm{~ms}$. It was the averaged motion of 28 times.

\section{Vibration of The Movable Part}

The simplified mechanical model of the movable part is proposed in Fig. 15 considering the compliance of the structure. The elastic connection between the steel hemisphere and the washer was indicated by the spring. When the Rayleigh wave is excited, the driving force is given to the silicon slider. The silicon slider is driven to the direction to compress the spring. The compressed spring thrusts the linear guide stage in the meanwhile. After the Rayleigh wave passed away, there is no driving force to the silicon slider and then the silicon slider is thrust back by the elastic force of the compressed spring. In this way, the slider part, namely the silicon slider and the glued hemisphere, starts vibration according to the mass of the slider part and the elastic constant of the spring.

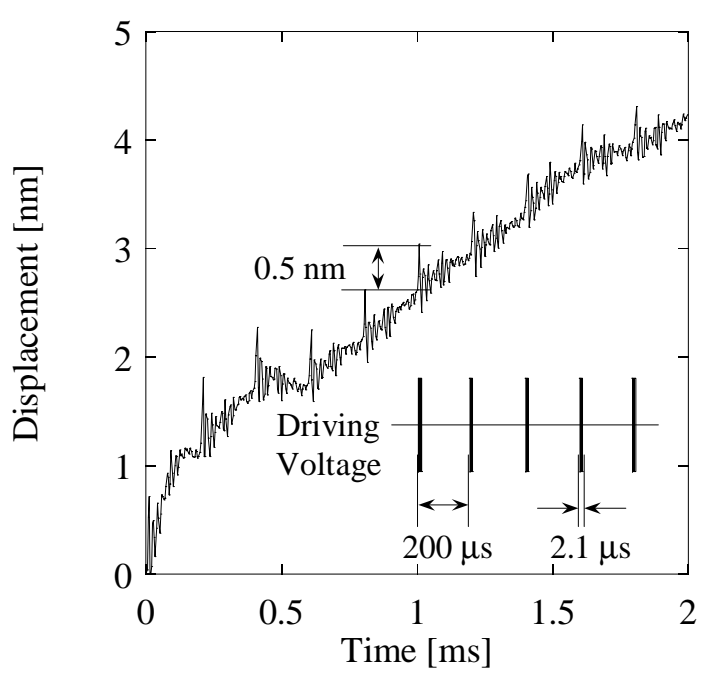

Fig.14 The stepping drive motion of $1 \mathrm{~nm}$; the driving voltage was 100 Veak, the pre-load was $30 \mathrm{~N}$, and the driving waves were 20 cycles. This friction driven displacement was about $0.5 \mathrm{~nm}$. The burst period was $0.2 \mathrm{~ms}$. It was the averaged motion of 30 times.

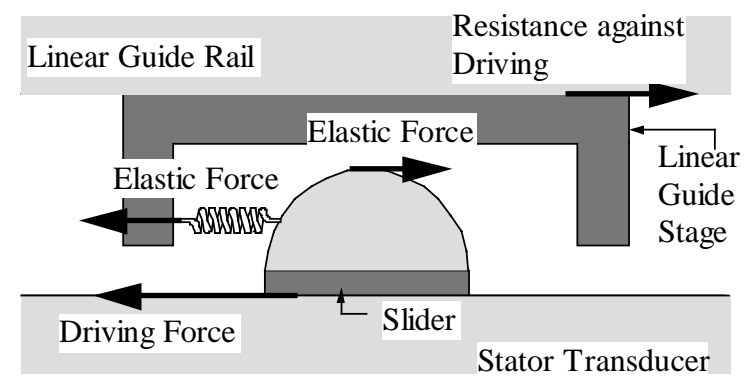

Fig.15 The simplified mechanical model of the movable part. 
On the other hand, the linear guide stage thrusts by the inertia that is given by the first movement of the driven slider part. As previously indicated, the mass of the linear guide stage was $9.42 \mathrm{~g}$ and the mass of the slider part, the slider and the glued hemisphere, was $0.89 \mathrm{~g}$. The mass of the linear guide part was about 10 times larger than the slider part. Hence, the linear guide stage does not vibrate as the slider part does. The linear guide stage responds slower to the elastic force of the compressed spring.

\section{Conclusion}

The nanometer stepping drives of the surface acoustic wave motor were examined. The finest stepping drive of the experimental setup was the $2 \mathrm{~nm}$ step motion. Although, the sub-nanometer step motion masked with the vibration is indicated. Then reduction of the vibration due to the compliance of the structure is required. The rigid structure will perform a fine subnanometer stepping motion.

\section{Acknowledgement}

This work was supported by the Grant-in-Aid for the Development of Innovative Technology of the Ministry of Education, Culture, Sports, Science and Technology of Japan.

\section{References}

[1] S. Ueha and Y. Tomikawa, "Ultrasonic motorsTheory and Applications," Oxford, Clarendon Press, 1993.

[2] M. Kurosawa, M. Takahashi and T. Higuchi, "Ultrasonic Linear Motor Using Surface Acoustic Waves," IEEE Trans. Ultrason., Ferroelect., Freq. Cont., vol.43, no.5, pp.901-906, 1996.

[3] N. Osakabe, M. Chiba, M. K. Kurosawa and T. Higuchi, "Experimental Estimation of Surface Acoustic Wave Linear Motor," Proc. of Int. Conf. on Micromechatronics for Info. and Precis. Equip. Tokyo, pp.265-268, 1997.

[4] M. K. Kurosawa and T. Higuchi, "Surface Acoustic Wave Motor," Proc. of 3rd Int. Heintz Nixdorf Symp., Paderborn, Germany, pp.113-118, 1999.

[5] M. K. Kurosawa, "State-of-the-art surface acoustic wave linear motor and its future applications," Ultrasonics 38, pp.15-19, 2000.

[6] M. Takasaki, M. K. Kurosawa and T. Higuchi, "Optimum contact conditions for miniaturized Surface acoustic wave linear motor," Ultrasonics 38, pp.51-53, 2000.

[7] M. K. Kurosawa, H. Itoh, K. Asai, M. Takasaki and T. Higuchi, "Optimization of Slider Contact Face Geometry for Surface Acoustic Wave Motor," Proc. of Micro Electro Mechanical Systems, Interlaken, Suisse, pp.252-255, 2001. 